\title{
Commentary: Physical Approaches for the Treatment of Epilepsy: Electrical and Magnetic Stimulation and Cooling
}

\author{
Wolfgang Löscher, ${ }^{\dagger}{ }^{\dagger}$ Andrew J. Cole, ${ }^{*}$ and Michael J. McLean ${ }^{\|}$ \\ *Department of Pharmacology, Toxicology, and Pharmacy, University of Veterinary Medicine Hannover, Hannover D-30559, \\ Germany, ${ }^{\dagger}$ Center for Systems Neuroscience, Hannover, D-30559 Germany, ${ }^{\ddagger}$ Epilepsy Service, Massachusetts General Hospital, \\ Boston, Massachusetts 02114, "Vanderbilt University Medical Center, Department of Neurology, Nashville, Tennessee 37212
}

\begin{abstract}
Summary: Physical approaches for the treatment of epilepsy currently under study or development include electrical or magnetic brain stimulators and cooling devices, each of which may be implanted or applied externally. Some devices may stimulate peripheral structures, whereas others may be implanted directly into the brain. Stimulation may be delivered chronically, intermittently, or in response to either manual activation or computer-based detection of events of interest. Physical approaches may therefore ultimately be appropriate for seizure prophylaxis by causing a modification of the underlying substrate, presumably with a reduction in the intrinsic excitability of cerebral structures, or for seizure termination, by interfering with the spontaneous dis-
\end{abstract}

charge of pathological neuronal networks. Clinical trials of device-based therapies are difficult due to ethical issues surrounding device implantation, problems with blinding, potential carryover effects that may occur in crossover designs if substrate modification occurs, and subject heterogeneity. Unresolved issues in the development of physical treatments include optimization of stimulation parameters, identification of the optimal volume of brain to be stimulated, development of adequate power supplies to stimulate the necessary areas, and a determination that stimulation itself does not promote epileptogenesis or adverse long-term effects on normal brain function. Key Words: Brain stimulation, vagus nerve, hypothermia, neuromodulation, seizures.

\section{INTRODUCTION}

At least $30 \%$ of patients with epilepsy have uncontrolled seizures, despite treatment with anti-epileptic drugs (AEDs). In addition, adverse effects of AEDs compromise the quality of life of many patients whether or not seizures are controlled adequately or may even prevent titration to effective doses. Carefully selected AEDresistant patients may benefit from surgical resection, but epilepsy surgery has risks and costs, and many forms of AED-resistant epilepsy are not surgically amenable. The adverse seizure and neurobehavioral prognosis in patients with AED-resistant epilepsy provides ample justification to search for alternative treatments. Physical approaches, including brain stimulation and cooling, are among the most promising experimental alternative therapies.

During the Workshop on "New Horizons in the Development of Antiepileptic Drugs: Non-Traditional

Address correspondence and reprint requests to: Wolfgang Löscher, M.D., Department of Pharmacology, Toxicology and Pharmacy, University of Veterinary Medicine, Bünteweg 17, Hannover, D-30559 Germany. E-mail: wolfgang.loescher@tiho-hannover.de.
Approaches to Treat Epilepsy" (March 5-7, 2008, Clontarf Castle, Dublin, Ireland), one session focused on electrical and magnetic stimulation and cooling as experimental alternative therapies for AED-resistant epilepsy. There were five talks within this topic, followed by "pro" and "con" summaries by M. J. McLean and W. Löscher, and general discussion. The session was chaired by N. Delanty and A. Cole. In the following, we will briefly discuss the alternative therapies reviewed in this session.

\section{ELECTRICAL BRAIN STIMULATION}

Neurostimulation-based treatments such as vagus nerve stimulation (VNS), deep brain stimulation (DBS), and responsive neurostimulation (RNS), have gained increasing attention in recent years. ${ }^{1,2}$ Underlying all these techniques is the idea that extrinsic stimulation can reduce hyperexcitability in seizure-generating networks, perhaps by interrupting the build-up to the ictal transition. VNS, the only approved stimulation therapy for epilepsy, has been licensed for nearly a decade in several countries 
as an adjunctive therapy for refractory epilepsy, ${ }^{3,4}$ and for several years as a treatment for medication-resistant depression. Depression is a frequent comorbid condition with epilepsy, ${ }^{5}$ and its presence before seizure onset predicts refractoriness of partial seizures. ${ }^{6}$ Some AEDs may exacerbate depression and increase the risk of suicide. $^{7}$ Thus, VNS, alone or in combination with AEDs, offers the possibility of improving the quality of patients' lives by improving seizure control, minimizing the systemic load of AEDs, and improving mood. Coexistence of depressive symptoms may be a factor in selecting VNS treatment. ${ }^{8}$ Despite the hopes and expectations these prospects raise, efficacy of VNS has been limited.

VNS uses a pulse generator that transmits impulses to the left vagus nerve via an implantable electrode and can be implanted by surgeons familiar with the anatomy of the cervical vagus nerve. Afferent fibers of the vagus nerve project to several brain regions, including the thalamus, amygdala, and forebrain, through the nucleus tractus solitarius and the medullary reticular formation. Electrical stimulation of the vagus nerve is thought to affect pathogenetic mechanisms, which are thought to be involved in the generation and propagation of seizures ${ }^{3}$ and depression. ${ }^{6,7}$ However, the exact mechanisms by which VNS exerts its effects are unknown. As an adjunctive therapy in AED-resistant patients, VNS typically reduces seizure frequency in "VNS responders," although few patients become seizure-free. ${ }^{3}$ The efficacy of VNS in reducing the frequency or severity of seizures is highly variable, and no clear predictive factors for responders have been identified. Even though the pulse parameters can be varied widely, protocols that treat seizure types selectively have not been established. Early VNS treatment in the course of pharmacoresistant epilepsy may improve the benefit. ${ }^{9}$ Several controlled trials have assessed the efficacy of VNS in patients with epilepsy. ${ }^{2}$ Because patients can sense VNS stimulation, traditional blinded, placebo-controlled trials have not been practical. Instead, to obtain "controlled" data, a dose-response design with "high" and "low" stimulation was adopted, showing 25 to $30 \%$ mean reduction in seizure frequency for the high-stimulation group versus 6 to $15 \%$ for the low-stimulation group. ${ }^{2}$ Thus, the clinical efficacy of VNS is limited, and at best comparable with adjunctive therapy with novel AEDs. However, individual patients may benefit from VNS, particularly because the therapeutic efficacy is sustained and VNS is generally welltolerated. There appears to be a delay before the maximal anticonvulsant effect of VNS develops, a phenomenon that is not understood. If a reduction in seizure frequency is not observed after a prolonged period (e.g., 18 months as recommended by Cyberonics, Inc.), the device should be deactivated and removed.

DBS is an investigational approach that requires intracranial (stereotaxic) surgery, and thus is more invasive than VNS. ${ }^{1,2}$ DBS has been used extensively as a treatment for movement disorders, such as Parkinson's disease. ${ }^{10}$ Various brain targets for DBS in epilepsy have been investigated, including thalamus, hippocampus, and subthalamic nucleus ${ }^{11,12}$ with the idea of stimulating more discreet networks that may be more closely linked to the seizure generator than the more widespread effects of VNS. ${ }^{13}$ Several results have been encouraging, but not conclusive. As with other investigational therapies, the efficacy of DBS was higher in open trials than in the few available controlled trials. ${ }^{2}$ The best structure to stimulate and the most effective stimuli to use are unknown as yet. The risks of DBS demands careful consideration of its value on an individual basis. Electrode implantation in the brain has been associated with a 5\% risk for intracranial hemorrhage and $5 \%$ for infection. ${ }^{1}$

RNS is a more recent concept based on the idea that spontaneous epileptic activity can be terminated by inhibitory polarization produced by slow intermittent electrical stimulation applied to the region of onset. ${ }^{14,15} \mathrm{Po}-$ tential advantages of RNS include the ability to be applied to bilateral or multiple foci, eloquent cortex that can not be excised, pathways of seizure propagation or pathways with global modulatory effects à la VNS, with or without tailored AED therapy. ${ }^{14}$ Seizure-prediction algorithms could be used, even though only seizure detection algorithms have been studied so far. ${ }^{14}$ Combining other physical approaches, such as cooling, with RNS is another prospect that comes to mind. ${ }^{16}$ In contrast to DBS, the electrical stimulation is not continuously applied, but only acutely after detection of seizure onset. The ultimate goal of such a closed-loop feedback system for seizure detection and brain stimulation is the creation of a totally implantable device. The optimal location (deep brain or cortical), characteristics of the stimulation (frequency, current, duration), and threshold for event detection and stimulation are still to be determined. In a feasibility study of 65 patients, the responder rate was $43 \%$ for complex partial seizures and $35 \%$ for partial (simple and complex) and secondarily generalized tonicclonic seizures. ${ }^{15}$ A large, controlled clinical trial is currently underway. Controls will receive sham stimulation via implanted electrodes. Risks of RNS are comparable to those of DBS. Furthermore, both DBS and RNS may be associated with micro-lesions and mechanical effects due to electrode implantation, which by themselves may affect seizure activity.

\section{Transcranial magnetic and direct current stimulation}

In comparison to VNS, DBS, and RNS, transcranial magnetic stimulation and transcranial direct current stimulation (tDCS) are the simplest and least invasive approaches to brain stimulation for epilepsy. ${ }^{17-19}$ Transcranial magnetic stimulation with either a hand-held magnet or a frame that can be aligned to predetermined coordi- 
nates is used as a diagnostic tool in patients, particularly to measure parameters related to cortical excitatory and inhibitory function. ${ }^{19}$ Low-frequency, repetitive transcranial magnetic stimulation (rTMS) reduces motor cortex excitability in humans, and a number of studies have explored this technique as a therapeutic approach in patients with AED-resistant epilepsy. ${ }^{2}$ Results have been equivocal, with small and transient responses. A small, controlled study with rTMS in patients with mesial temporal or neocortical localization-related epilepsy did not demonstrate any significant effect on seizure frequency, although actively treated patients had a $16 \%$ (nonsignificant) mean reduction in seizure frequency, but only in the first two weeks after stimulation. ${ }^{20}$ There was a trend toward a greater effect in patients with neocortical rather than mesial temporal foci, suggesting that mesial temporal structures probably lie too deep beneath the surface for effective stimulation by rTMS. ${ }^{2}$ In another study with slow rTMS $(0.3 \mathrm{~Hz})$ significant reduction of EEG epileptiform activity was observed in one third of subjects, supporting a biological effect; however, seizure frequency was not significantly reduced. ${ }^{21}$ A few rTMS experiments have been performed in animal models of epilepsy (e.g., Ebert and Ziemann ${ }^{22}$ ), but the need to immobilize the animals during stimulation and the local heating associated with rTMS have prevented any meaningful conclusions.

More recently, tDCS has been proposed to constitute a simple and effective means for brain stimulation in patients with epilepsy. ${ }^{17}$ tDCS involves the application of low currents to the scalp via cathodal or anodal electrodes and has been shown to affect a range of motor, somatosensory, visual, affective, and cognitive functions. ${ }^{18}$ A controlled, clinical trial of cathodal tDCS in patients with malformations of cortical development and refractory epilepsy showed a nonsignificant trend toward decrease in seizure frequency after active versus sham DC treatment. ${ }^{23}$ In an animal model of neocortical epilepsy, cathodal tDCS induced anticonvulsant effects, albeit of lower magnitude than those induced by the AED valproate. $^{24}$

\section{Cooling}

An attractive alternative physical approach for terminating and perhaps preventing focal seizures is local (focal) cooling. ${ }^{16}$ The potential clinical utility of therapeutic cooling or hypothermia for neurological disorders associated with neuronal injury or excessive electrical activity, including traumatic brain injury, stroke, asphyxia, and epilepsy, has been discussed for decades, but there has been renewed interest in this approach in recent years. Of particular interest has been the potential of hypothermia to be neuroprotective, which has been demonstrated in a variety of preclinical and clinical studies. $^{25,26}$ Different techniques for therapeutic cooling have been used both preclinically and clinically, including surface cooling, endovascular cooling, and direct brain cooling, such as focal cooling using a piezoelectric device. $^{16,27}$ In vitro and in vivo experimental epilepsy studies have demonstrated that cooling diminishes paroxysmal bursting and can reduce or stop seizure activity. ${ }^{16}$ External cooling was reported to terminate AED-refractory status epilepticus in two clinical investigations and one study in a rat model. ${ }^{28,29}$ In patients, direct cooling of the brain under anesthesia was described to block seizures. ${ }^{30,31}$ Transient head cooling in infants with hypoxic-ischemic encephalopathy increased survival and reduced the severity of EEG abnormalities. ${ }^{32}$ However, such techniques of therapeutic cooling are not suitable for chronic application in patients with AED-resistant epilepsy. In a series of experiments, Rothman et al. ${ }^{16}$ have developed and evaluated thermoelectric devices that may be applicable for the chronic therapy of partial epilepsy. This technique suppresses fully kindled seizures in awake, freely moving rats ${ }^{33}$ without producing any signs of neuronal injury. ${ }^{34}$ The ultimate expectation of Rothman et al. ${ }^{16}$ is an implantable device that would allow cooling at a site of seizure initiation under closed-loop feedback control, similar to the design used for RNS. Despite the optimism generated by preclinical and uncontrolled clinical studies in different neurological diseases, there is currently no evidence from randomized controlled trials to support the routine use of cooling in traumatic brain injury, stroke, or epilepsy. ${ }^{26}$

\section{CONCLUSIONS}

Physical (nonpharmacological) approaches offer interesting alternatives for the treatment of AED-resistant epilepsies. Table 1 lists relative advantages and disadvantages of each of the approaches discussed based on currently available data and experience. One of these approaches, VNS, has been used in tens of thousands of patients with epilepsy since its approval, whereas other physical techniques are still at an experimental stage. Overall, the anticonvulsant efficacy that can be obtained with physical approaches is relatively low and comparable with the moderate reduction in seizure frequency that is typically seen with adjunctive therapy with new AEDs in patients with intractable epilepsy. Furthermore, in contrast to drug trials, traditional placebo-controlled trials are not practical with devices such as VNS, DBS, RNS, or cooling, due to the patients' ability to perceive when stimulation or cooling is "on," In some studies, sham controls with the same invasive procedure, but no stimulation have been used, but this does not resolve the problem of sensing the stimulus. Because of the difficulty of blinding the subjects, trials with high- and lowstimulation parameters may be useful, as performed with VNS. The phenomenon of "reversion to the mean" may 
Table 1. Potential Advantages and Disadvantages of Proposed Physical Treatment Approaches*

\begin{tabular}{|c|c|c|c|c|c|c|c|}
\hline & Invasiveness & Portability & $\begin{array}{l}\text { Potential to } \\
\text { Treat Multiple } \\
\text { Foci }\end{array}$ & Efficacy & Tolerability & Potential Limitations & Potential Advantages \\
\hline VNS & ++ & ++++ & - & ++ & +++ & $\begin{array}{l}\text { Broad range of stimulation parameters possible, but } \\
\text { values for specific seizure types not completely } \\
\text { explored or understood. }\end{array}$ & $\begin{array}{l}\text { - Approved by regulatory agencies in } \\
\text { many countries. } \\
\text { - Conveniently programmable. } \\
\text { - Seizure freedom possible for some. }\end{array}$ \\
\hline DBS & +++ & ++++ & - & ++ & ++++ & $\begin{array}{l}\text { Sterotaxic electrode placement necessary. } \\
\text { Risk of hemorrhage and infection. } \\
\text { Determining the best site for electrode placement is } \\
\text { complex, invasive, and largely empirical. }\end{array}$ & $\begin{array}{l}\text { - Stimulation of discreet networks close } \\
\text { to seizure generator is possible. } \\
\text { - Multiple possible implantation sites } \\
\text { affords some individualization. }\end{array}$ \\
\hline RNS & +++ & ++++ & +++ & $?$ & ++++ & $\begin{array}{l}\text { Efficacy of treatment triggered by seizure initiation } \\
\text { (vs seizure prediction) remains to be determined. } \\
\text { Seizure freedom not observed in proof-of-concept } \\
\text { study. } \\
\text { Electrode placement is critical. } \\
\text { Microlesions, mechanical effects, and electrolysis } \\
\text { near electrodes can affect seizure frequency. }\end{array}$ & $\begin{array}{l}\text { - Configurable detection and } \\
\text { stimulation algorithms allow } \\
\text { individualization of therapy. } \\
\text { - Programmable restimulations for } \\
\text { persistent seizure activity. } \\
\text { - Adjustment of stimulus parameters } \\
\text { minimizes proconvulsant effects and } \\
\text { possibility of microlesions. } \\
\text { - Large multicenter controlled clinical } \\
\text { trial in progress. } \\
\text { - Potential combination with other } \\
\text { physical approaches, e.g. cooling, at } \\
\text { electrode site. }\end{array}$ \\
\hline rTMS & - & - & ++ & $?$ & ++ & $\begin{array}{l}\text { Available technology limits stimulation frequency. } \\
\text { Depth reached by effective field is limited, thereby } \\
\text { limiting efficacy and utility. }\end{array}$ & $\begin{array}{l}\text { - Intermittent or daily stimulation may } \\
\text { be effective, as for bipolar disorder, } \\
\text { an indication approved by regulatory } \\
\text { agencies in some countries. }\end{array}$ \\
\hline $\mathrm{tDCS}$ & - & + & - & $?$ & ++++ & $\begin{array}{l}\text { The range of useful current strengths is likely to be } \\
\text { limited. } \\
\text { Depth of current spread is likely to be limited or } \\
\text { unknown. } \\
\text { Optimum treatment protocols have not been determined. }\end{array}$ & $\begin{array}{l}\text { - Direct hyperpolarizing current is not } \\
\text { proconvulsant. }\end{array}$ \\
\hline $\begin{array}{l}\text { Focal } \\
\text { Cooling }\end{array}$ & +++ & + & +++ & $?$ & $?$ & $\begin{array}{l}\text { Excessive focal cooling could produce tissue damage. } \\
\text { Implantable (e.g., piezoelectric) devices useful for } \\
\text { clinical trials in man are limited. } \\
\text { Determination of temperature at cooling site is essential } \\
\text { for optimization and is difficult with extracranial } \\
\text { devices. } \\
\text { Systemic effects (e.g., on the heart, limit external } \\
\text { cooling). }\end{array}$ & $\begin{array}{l}\text { - Chronic focal cooling with } \\
\text { electrodes of the type used in RNS } \\
\text { may be possible. } \\
\text { - Combination with other physical } \\
\text { techniques, e.g. RNS, may be } \\
\text { possible. } \\
\text { - Cooling may be neuroprotective. }\end{array}$ \\
\hline
\end{tabular}

,,++++++ , and ++++ represents relative value. - indicates noninvasive or not available. ? indicates no data available.

DBS = deep brain stimulation; RNS = responsive neurostimulation; rTMS = repetitive transcranial magnetic stimulation; tDCS = transcranial direct current stimulation; VNS = vagus nerve stimulation.

*All physical approaches below can be combined with antiepileptic drug therapy. Stimulation techniques have potential proconvulsant effects that can be addressed by varying stimulation parameters. 
Table 2. Common Problems in Designing Trials for Assessing the Efficacy of Physical Approaches

- Blinding may be difficult or impossible

- Relationship of implant localization and zone of epileptogenesis may be difficult to establish

- Risks of infection, injury, and potential epileptogenisis are unknown

- Availability of well established alternative treatments for focal epilepsy negatively impact recruitment

- Choice of stimulation parameters is often based on little empiric data

- Chronic intermittent stimulation may lead to tachyphylaxis and/or proconvulsant effects that confound interpretation of study results

- Potential latency to full effect may lead to falsenegative results

produce an additional bias. ${ }^{2}$ Patients are more likely to enter an experimental trial when their seizure frequency is high, leading to the risk that decline in frequency during the trial, due to random variation, might be misinterpreted as a therapeutic effect. This risk may be higher for studies involving invasive procedures or surgery than AEDs. ${ }^{2}$ This by no means proves that physical approaches are not effective, but emphasizes the importance of randomized clinical trials to establish the efficacy and safety of these methods. Table 2 lists some of the potential difficulties encountered in conducting trials of physical approaches to epilepsy treatment.

\section{REFERENCES}

1. Theodore WH, Fisher RS. Brain stimulation for epilepsy. Lancet Neurol 2004;3:111-118.

2. Theodore WH, Fisher R. Brain stimulation for epilepsy. Acta Neurochir Suppl 2007;97:261-272.

3. Groves DA, Brown VJ. Vagal nerve stimulation: a review of its applications and potential mechanisms that mediate its clinical effects. Neurosci Biobehav Rev 2005;29:493-500.

4. Boon P, De H, V, Vonck K, Van Roost D. Clinical experience with vagus nerve stimulation and deep brain stimulation in epilepsy. Acta Neurochir Suppl 2007;97:273-280.

5. Milby $\mathrm{AH}$, Halpern $\mathrm{CH}$, Baltuch $\mathrm{GH}$. Vagus nerve stimulation for epilepsy and depression. Neurotherapeutics 2008;5:75-85.

6. Kanner AM. Depression in epilepsy: a complex relation with unexpected consequences. Curr Opin Neurol 2008;21:190-194.

7. Miller JM, Kustra RP, Vuong A, Hammer AE, Messenheimer JA. Depressive symptoms in epilepsy: prevalence, impact, aetiology, biological correlates and effect of treatment with antiepileptic drugs. Drugs 2008;68:1493-1509.

8. Brodtkorb E, Mula M. Optimizing therapy of seizures in adult patients with psychiatric comorbidity. Neurology 2006;67(suppl 4):S39-S44.

9. Helmers SL, Griesemer DA, Dean CJ, et al. Observations on the use of vagus nerve stimulation earlier in the course of pharmacoresistant epilepsy: patients with seizures for six years or less. The Neurologist 2003;9:160-164.

10. Breit S, Schulz JB, Benabid AL. Deep brain stimulation. Cell Tissue Res 2004;318:275-288.

11. Vonck K, Boon P, Van Roost D. Anatomical and physiological basis and mechanism of action of neurostimulation for epilepsy. Acta Neurochir Suppl 2007;97:321-328.
12. Halpern CH, Samadani U, Litt B, Jaggi JL, Baltuch GH. Deep brain stimulation for epilepsy. Neurotherapeutics 2008;5:59-67.

13. Li Y, Mogul DJ. Electrical control of epileptic seizures. J Clin Neurophysiol 2007;24:197-204.

14. Morrell M. Brain stimulation for epilepsy: can scheduled or responsive neurostimulation stop seizures? Curr Opin Neurol 2006; 19:164-168.

15. Sun FT, Morrell MJ, Wharen Jr. RE. Responsive cortical stimulation for the treatment of epilepsy. Neurotherapeutics 2008;5: $68-75$.

16. Rothman SM, Smyth MD, Yang XF, Peterson GP. Focal cooling for epilepsy: an alternative therapy that might actually work. Epilepsy Behav 2005;7:214-221.

17. Paulus W. Transcranial direct current stimulation (tDCS). Suppl Clin Neurophysiol 2003;56:249-254.

18. Been G, Ngo TT, Miller SM, Fitzgerald PB. The use of tDCS and CVS as methods of non-invasive brain stimulation. Brain Res Rev 2007;56:346-361.

19. Chen R, Cros D, Curra A, Di L, V, Lefaucheur JP, Magistris MR, et al. The clinical diagnostic utility of transcranial magnetic stimulation: report of an IFCN committee. Clin Neurophysiol 2008; 119:504-532.

20. Theodore WH, Hunter K, Chen R, Vega-Bermudez F, Boroojerdi $\mathrm{B}$, Reeves-Tyer $\mathrm{P}$, et al. Transcranial magnetic stimulation for the treatment of seizures: a controlled study. Neurology 2002;59:560562.

21. Cantello R, Rossi S, Varrase C, et al. Slow repetitive TMS for drug-resistant epilepsy: clinical and EEG findings of a placebocontrolled trial. Epilepsia 2007;48:366-374.

22. Ebert U, Ziemann U. Altered seizure susceptibility after highfrequency transcranial magnetic stimulation in rats. Neurosci Lett 1999;273:155-158.

23. Fregni F, Thome-Souza S, Nitsche MA, Freedman SD, Valente KD, Pascual-Leone A. A controlled clinical trial of cathodal DC polarization in patients with refractory epilepsy. Epilepsia 2006; 47:335-342.

24. Liebetanz D, Klinker F, Hering D, et al. Anticonvulsant effects of transcranial direct-current stimulation (tDCS) in the rat cortical ramp model of focal epilepsy. Epilepsia 2006;47:1216-1224.

25. Gunn AJ, Gunn TR. The 'pharmacology' of neuronal rescue with cerebral hypothermia. Early Human Development 1998;53:19-35.

26. Polderman KH. Induced hypothermia and fever control for prevention and treatment of neurological injuries. Lancet 2008;371: 1955-1969.

27. Hemmen TM, Lyden PD. Induced hypothermia for acute stroke. Stroke 2007;38(Suppl 2):794-799.

28. Vastola EF, Homan R, Rosen A. Inhibition of focal seizures by moderate hypothermia. A clinical and experimental study. Arch Neurol 1969;20:430-439.

29. Schmitt FC, Buchheim K, Meierkord H, Holtkamp M. Anticonvulsant properties of hypothermia in experimental status epilepticus. Neurobiol Dis 2006;23:689-696.

30. Sartorius CJ, Berger MS. Rapid termination of intraoperative stimulation-evoked seizures with application of cold Ringer's lactate to the cortex. Technical note. J Neurosurg 1998;88:349-351.

31. Karkar KM, Garcia PA, Bateman LM, Smyth MD, Barbaro NM, Berger M. Focal cooling suppresses spontaneous epileptiform activity without changing the cortical motor threshold. Epilepsia 2002;43:932-935.

32. Gluckman PD, Wyatt JS, Azzopardi D, et al. On behalf of the CoolCap Study Group. Selective head cooling with mild systemic hypothermia after neonatal encephalopathy: multicentre randomized trial. Lancet 2005;365:663-670.

33. Burton JM, Peebles GA, Binder DK, Rothman SM, Smyth MD. Transcortical cooling inhibits hippocampal-kindled seizures in the rat. Epilepsia 2005;46:1881-1887.

34. Yang XF, Kennedy BR, Lomber SG, Schmidt RE, Rothman SM. Cooling produces minimal neuropathology in neocortex and hippocampus. Neurobiol Dis 2006;23:637-643. 